\title{
Identification of Bacterial Diseases in Rice Plants Leaves by the Use of Spectroscopic Imaging
}

\author{
Mama Sangare $^{1}$, C. Tekete ${ }^{1}$, O. K. Bagui ${ }^{2}$, A. Ba ${ }^{1} \&$ J. T. Zoueu ${ }^{2}$ \\ ${ }^{1}$ Laboratoire d'optique, de Spectroscopie et des Sciences de l'Atmosphere, University of Sciences, Technique and \\ Technology Bamako, Mali \\ ${ }^{2}$ Laboratoire d'Instrumentation Image et Spectroscopie, Institut National Polytechnique Felix Houphouët-Boigny \\ Yamoussoukro, Cote d'Ivoire \\ Correspondence: J. T. Zoueu, Laboratoire d'Instrumentation Image et Spectroscopie, Institut National \\ Polytechnique Felix Houphouët-Boigny Yamoussoukro, Cote d'Ivoire. Tel: 225-3064-6684. E-mail: \\ Jeremie.zoueu@inphb.edu.ci
}

Received: September 15, 2015 Accepted: September 24, $2015 \quad$ Online Published: October 24, 2015

doi:10.5539/apr.v7n6p61 URL: http://dx.doi.org/10.5539/apr.v7n6p61

\begin{abstract}
Rice is staple in the African habitats menu. Bacterial wilt (BLB) and leaf streak (BLS) are some of the phytopathological diseases which restrain rice production around the world. In this paper, multi-spectral and multimodal imaging techniques have been developed to characterize the rice leaves with symptoms of bacterial wilt (BLB) and leaf streak (BLS), and to provide information on their effects, in order to reduce their spread. First, we recorded microscopic and spectroscopic images of the samples using multimodal and multispectral microscope, with spectral region ranging from UV to NIR, for each mode. Then, we extracted the spectral footprints of the cells constituents, in transmission, reflection and scattering from the spectral images. Applying multivariate statistical analysis methods to this optical spectra allowed us to characterize the effect of bacterial rice leaves caused by Xanthomonas oryzae strains. The results of the proposed technique can be useful for easy identification of this type of infection, and can serve as routine approach in biochemical and agronomic laboratories.
\end{abstract}

Keywords: spectroscopic imaging, multispectral and multimodal microscopy, classification, rice, plant cell diseases, Xanthomonas oryzae

\section{Introduction}

Rice is the second most consumed cereal after maize in the world with a cultivated area of about 153 millions ha, a production of more than $608 \mathrm{Mt}$ and an average yield of $4.0 \mathrm{t} / \mathrm{ha}$. Rice occupies a prominent place in agriculture and food security maintaining. Unfortunately, rice has a hypersensitivity to phytopathogenic genus Xanthomonas oryzae pv. oryzae and Xanthomonas oryzae pv. oryzicola responsible for serious diseases such as bacterial wilt and leaf streak respectively. The yield losses of rice due to these diseases are enormous (Basso et al., 2011). In fact, to reduce the damage caused by these bacterial diseases, it is important to build capacity and improve knowledge about the relationship between the rice plants and the bacteria responsible for these pathologies. In West Africa, bacterial wilt was first reported in Mali in 1979 (Buddenhagen, Vuong, \& Ba, 1979); then in 1980 in Senegal, in 1981 in Burkina Faso and in Niger in 1983. In some West African countries (Burkina Faso, Niger and Mali), much damage has been produced by this devastating disease of rice. Many studies have also been conducted on these bacteria, particularly in Asia, exposing the losses they cause in rice production fields (Ou, 1985; Adhikari, Mew, \& Teng, 1994). In the Philippines, resistant varieties to certain strains of Xanthomonas oryzae pv. Oryzae have been developed (Mew, Vera Cruz, \& Reyes, 1992). In 2008, thirty Xoo breeds have been identified throughout the world (Kuljit \& al, 2008). Since the first observation and description of these plant diseases, no physical method has been established in West Africa in order to facilitate the fight against Xanthomonas oryzae pv oryzae (Srivastava \& Rao, 1964) and Xanthomonas oryzae pv. Oryzicola (Shekawat, Srivastava, \& Rao, 1969; Kihupi, 1997).

In this context, using the imaging spectroscopy as a diagnostic tool is an expanding area and its applications range from medical diagnostics to the environment including agriculture. Spectral imaging combines spectroscopy and imagery, to provide a new useful tool. It enables a measurement of a spectrum at each point of the image and provides a new insight on the specimen. This technique provides both spatial and spectral footprints resulting from the light-matter interaction. Practically, for a given wavelength, we obtain a multidimensional images resulting 
from two spatial dimensions that determine a point or pixel of the image, and a spectral dimension, which corresponds to the spectrum connected to the pixel. Among the others, some studies have been conducted (Olivier, Bagui, Zoueu, \& Wählby, 2015; Zoueu et al., 2009) using this technology, for the diagnosis of malaria using either spectroscopic imaging or statistical approach (Merdasa, Brydegaard, Svanberg, \& Zoueu, 2013; Bagui \& Zoueu, 2014; Bagui, Yavo, Tano, \& Zoueu, 2014). Recently, multi-spectral and multimodal imaging has been developed for the discrimination of different Ralstonia solanacearum subphenotypes in tobacco leaves and for the classification of certain symptoms African Mosaic Virus Infected Cassava Leaves (Sangare, Bagui, Traore, Babana, $\mathrm{Ba}, \&$ Zoueu, 2015). Much along these lines, in this work the use a multispectral and multimodal microscopy is proposed to distinguish the effect of the rice leaves bacterial wilt and leaf streak, and study simultaneously their influence during the process of photosynthesis. We believe that the results of this work will be helpful for researchers future investigations, and for producers, to improve the efficiency of their rice production.

\section{Materials and Methods}

\subsection{Biological Sample and Reproductive Symptoms of BLB and BLS on Rice}

The Xanthomonas team provided the samples; the team belongs to the Vegetable Biotechnology Unit (ULV) of the Applied Molecular Biology Laboratory (LBMA), of the Faculty of Science and Technology (FST). Rice leaves were collected with symptoms of bacterial wilt and streak leaf from three great centers of rice production in Mali (Baguineda, Selingué and Niono). One hundred and twenty samples were taken; strains of Xanthomonas oryzae pv. Oryzae and Xanthomonas oryzae pv. Oryzicola, responsible for these two diseases were isolated in LBMA on PSA medium (Peptone, Sucrose, Agar). This medium is prepared by combining in one liter of distilled water, 10g of peptone, $10 \mathrm{~g}$ of sucrose, $1 \mathrm{~g}$ of glutamic acid and $16 \mathrm{~g}$ bacto agar. The $\mathrm{pH}$ of the medium is adjusted to 7 . It is then autoclaved at $121^{\circ} \mathrm{C}$ for $20 \mathrm{~min}$. The multiplex PCR was used to confirm the infection. A strain of Xanthomonas oryzae pv. Oryzae (MAI1) was inoculated to Azucena's rice by cleavage of the upper end of the last two leaves « leaf clipping » with scissors, which were previously dipped in a calibrated inoculum to 6.108 bacteria / $\mathrm{ml}$ in order to reproduce the symptoms of bacterial wilt. This method was described by Kauffman et al. (1973). 24 hours before inoculation, the bacterial colonies of MAI1 were planted out again on PSA culture medium and incubated at $28^{\circ} \mathrm{C}$. The inoculum was prepared by suspension of these fresh bacterial colonies into sterile distilled water. Also, the strain of MAI10 of the collection of Xanthomonas oryzae pv. Oryzicola was also inoculated to plants of the variety of rice Kitaaké (japonica) by infiltration using a $1 \mathrm{ml}$ syringe following the method described by Reimer and Leach (1991). Both inocula are prepared according to the same method.Plants inoculated leaf clipping and infiltration are aged respectively 45 and 21 days. After three weeks of greenhouse production at the Faculty of Science and Technology, the symptoms of these two diseases were widely reproduced; the first leaves (infected and non-infected) were collected for microscopic examination, before their analysis with our imaging spectroscopy technology.

\subsection{Methods of Data Acquisition}

The experimental setup was described in (Zoueu et al., 2009; Merdasa, Brydegaard, Svanberg, \& Zoueu, 2013). It was built from a commercial microscope (Brunel Metallurgical microcope, model SP80), from which, the classical bright sources were removed and replaced for all modes, by a set of 13 LEDs, ranging from $375 \mathrm{~nm}$ to $940 \mathrm{~nm}$. The thirteen wavelengths were ordered as follows in nanomemters [375 400435470525590625660700750 $810850940]$. This enables the spectral coverage of this region with a mean step of $40 \mathrm{~nm}$. The initial mechanical oculars were substituted by a monochrome camera CMOS 12-bit (2592x1944, Guppy - 503B, Vision Allied Technology, with a sensor of MT9P031 micron / Aptina), with a pixel size of 2, $2 \mu \mathrm{m} \times 2,2 \mu \mathrm{m}$, which is used to acquire images.

The system acquires automatically a total of 39 spectral images (13 images by mode), for the same scene using a data acquisition card (NI-DAQ) coupled to a computer, which controls the intensities of the LEDs currents for fine adjustments of the parameters. In this work, we have imaged the rice leaves, infected by the strains of Xanthomonas oryzae pv, Oryzae and Xanthomonas oryzae pv. Oryzicola. Three modes (transmission, reflection and scattering) were used. For this spectral region, we have demonstrated that thirteen sources were sufficient to characterize the optical properties of these type of biological cells (Zoueu \& Zan, 2012). Transmission, reflection and scattering spectra (See Figure 3) were extracted from leaves (infected and non-infected) images.

\subsection{Data Analysis}

The analyzed data consist of three dimensions (spatial and spectral dimensions). The regions of interest correspond to selected microscopic images of rice leaves. In these regions, the objects analyzed and characterized, are the rice leaves exhibiting BLB and BLS symptoms. Objects are observed following 13 channels by mode. If $\mathrm{N}$ is the number of channels corresponding to spectral bands, each pixel observed along the $\mathrm{N}$ channels will be represented 
by a vector $\mathrm{X}$ belonging to a sub vector space of dimension $\mathrm{N}$. The data analysis method can be summarized by the flow chart below (see Figure 1). The data were analyzed by both PCA and K-means classification.

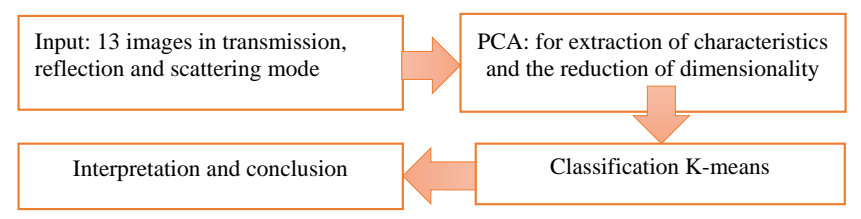

Figure 1. Schematic of data analysis procedure

\section{Results and Discussion}

\subsection{Results}

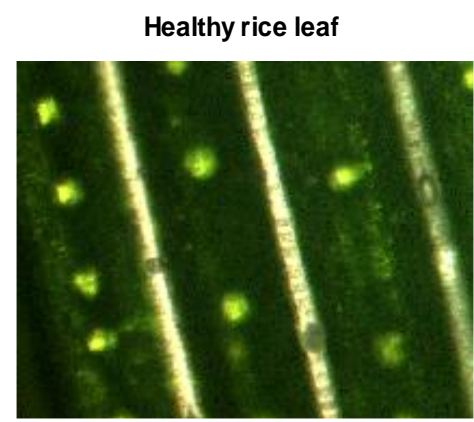

(a1)

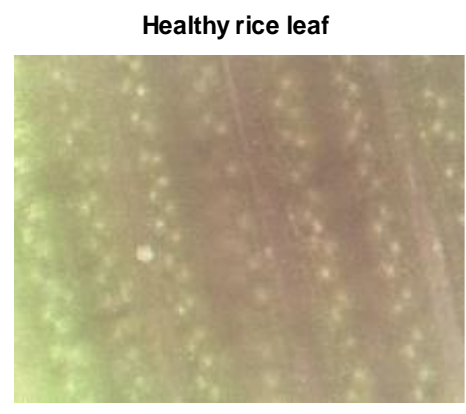

(b1)

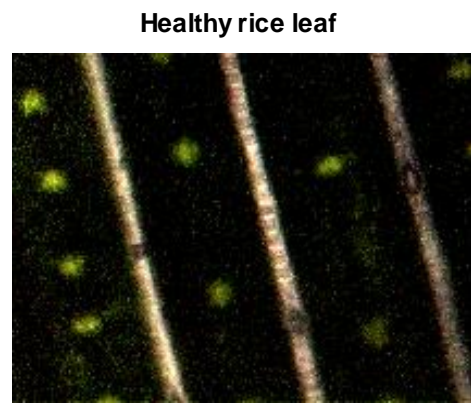

(c1)

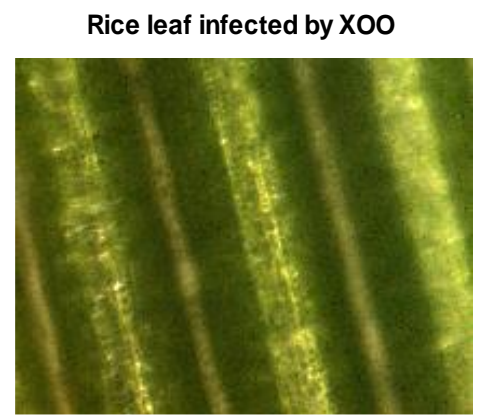

(a2)

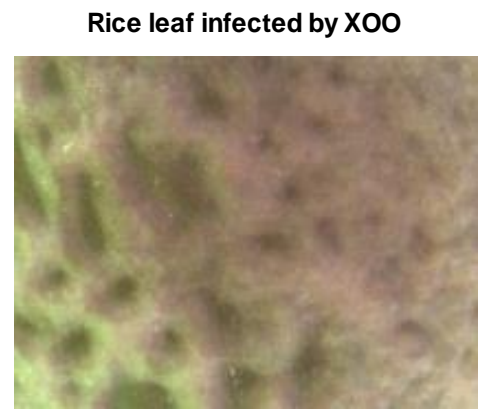

(b2)

Rice leaf infected by XOO

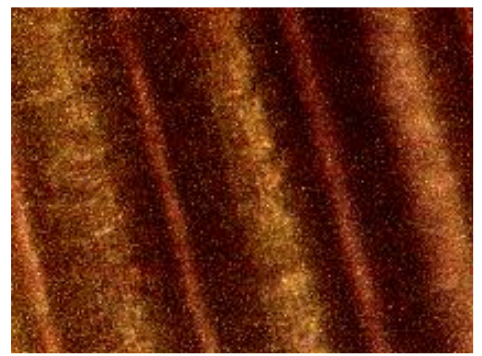

(c2)
Rice leaf infected by XOC

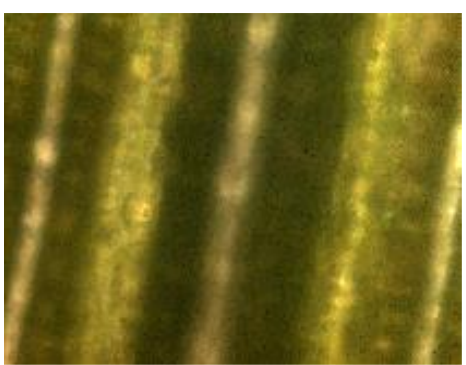

(a3)

Rice leaf infected by XOC

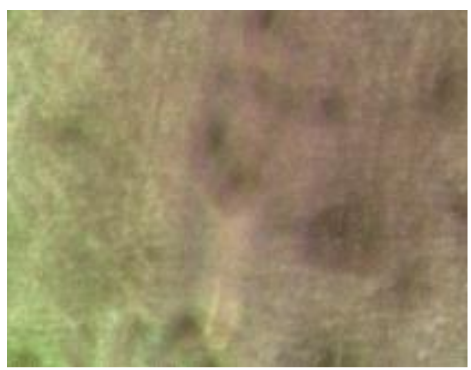

(b3)

Rice leaf infected by XOC

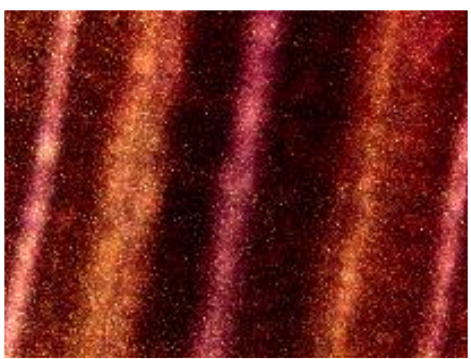

(c3)

Figure 2. Color sample images of healthy rice (a1, b1, and c1), infected by XOO (a2, b2, and c2), and infected by XOC (a3, b3, and c3) leaves, in transmission (a1, a2, and a3), reflection (b1, b2, and b3) and scattering (c1, c2, and c3) modes

We imaged 75 rice leaves ( 25 healthy leaves, 25 leaves infected by XOO and 25 leaves infected by XOC). The selected samples of these microscopic images exhibit various features in transmission, reflection and scattering modes (See Figures 2). 
The spectral signatures of healthy and infected rice leaves with the strains of XOO and XOC in the three modes may have redundancies in information. To reduce this redundancy by keeping the maximum of information, we will apply principal component analysis (PCA) method (Abdi and Williams, 2010). By applying PCA we have condensed the data for each vector $\mathrm{X}$ into new groups so that they would not present any correlation between them; they are also ordered in terms of the percentage of variance brought by each component. Thus, the first principal component contains information related to the maximum variance, the second contains information related to the following variance. The process is repeated up to the $\mathrm{n}^{\text {th }}$ principal component. The loss of information decreases from one stage to the next. So we obtain the principal component $Y_{i}$ with $i=1, \ldots$, I and $I \leq 13$. We have:

$$
Y_{i}=\sum_{\mathrm{j}=\mathrm{i}}^{\mathrm{N}}\left[V_{j} \cdot\left(\mathrm{X}_{\mathrm{i}}^{\mathrm{j}}-\overline{\mathrm{X}}_{\mathrm{i}}\right)\right]
$$

$\overline{\mathrm{X}}_{\mathrm{i}}$ : Represents the averages of each vector.

These averages are used to calculate covariance and correlation between variables $\mathrm{X}_{\mathrm{i}}$ and $\mathrm{X}_{\mathrm{j}}(\mathrm{i}=\mathrm{j})$;

$V_{j}$ : Represents proper vectors associated with proper values calculated from the polynomial of the matrix of covariance $\mathrm{S}$ :

$$
\mathrm{S}=\left\{\operatorname{Cor}\left(\mathrm{X}_{\mathrm{i}}, \mathrm{X}_{\mathrm{j}}\right)\right\}_{13 \times 13}
$$
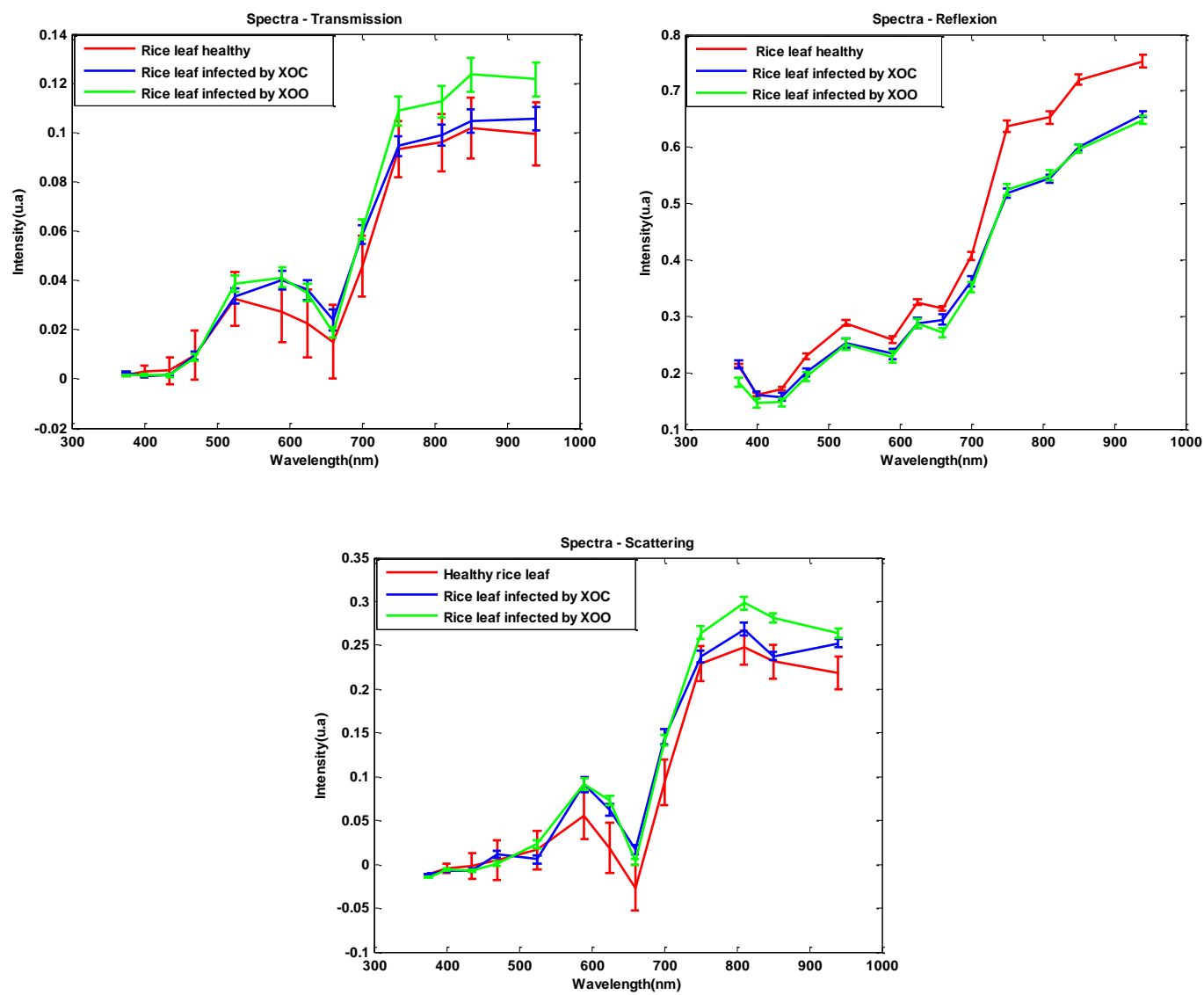

Figure 3. Comparison between healthy, infected by XOO and XOC leaves spectra, in transmission (a), reflection (b) and scattering (c) modes

However, the quality of the estimates, which led PCA, depends on the choice of the number of components retained to reconstruct the data, or the dimension of the subspace representation. So we have used the Eboulis criterion (Abdi \& Williams, 2010) with a graph presenting the decreasing of the proper values, and reducing the number of dimensionality.

The technique consists in searching for a potential "elbow" in the graph for each mode and keeping only the proper values up to this elbow (Figure 4) for the selection of principal components. 


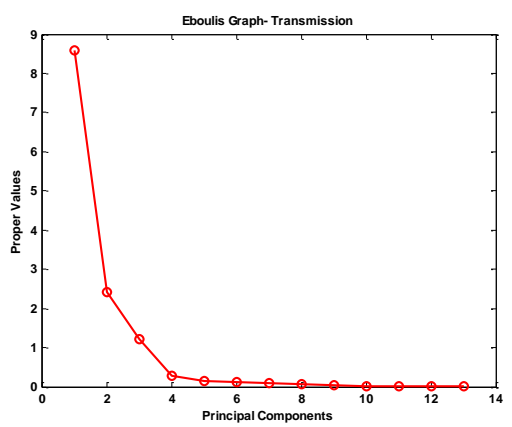

(a)

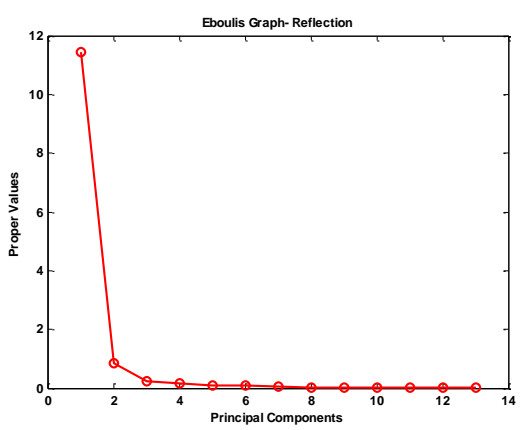

(b)

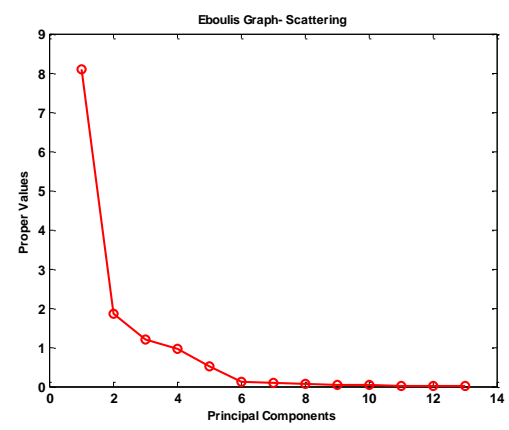

(c)

Figure 4. Eboulis representation of rice leaves in transmission (a), reflection (b) and scattering (c) modes According to the Eboulis graph:

- Components 2 and 3 can better describe our data in transmission;

- The components 1 and 2 can better describe our data in reflection mode;

- $\quad$ The components 1 and 3 also better describe our data in scattering mode.

- The projection of transmission, reflection and scattering mode in two-dimensional space of these principal components retained gives us the Figure 5.

Two or three classes are formed according to these projections in the three geometries. Healthy rice leaves form one class, but the two leaves infected by XOO and XOC seem to form one class together or two classes if the infected leaves are considered to be in the same class. The results of our principal component analysis are difficult to interpret physically because certain points have a great dispersion compared to elements of their class (Turler, 2006). So we made recourse to a cluster analysis methods: K-means classification. This method were applied to the data after the PCA. Figure 6 gives the results after application of the K-means classification.

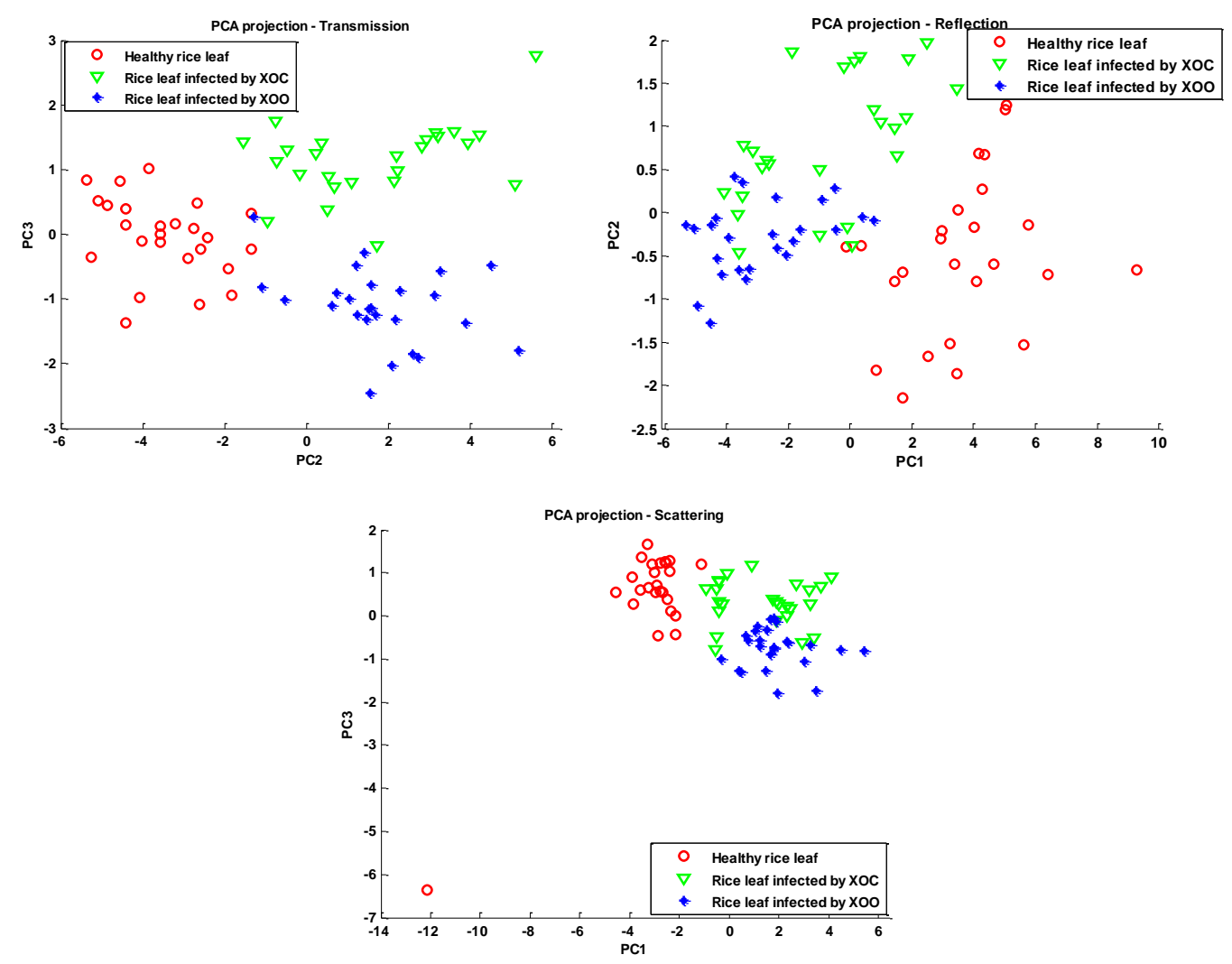

Figure 5. Comparative projection of healthy, infected by XOO and XOC leaves in transmission mode using PC2 and PC3 (a) reflection mode using PC1 and PC2 (b) and scattering mode using PC1 and PC3 (c) 

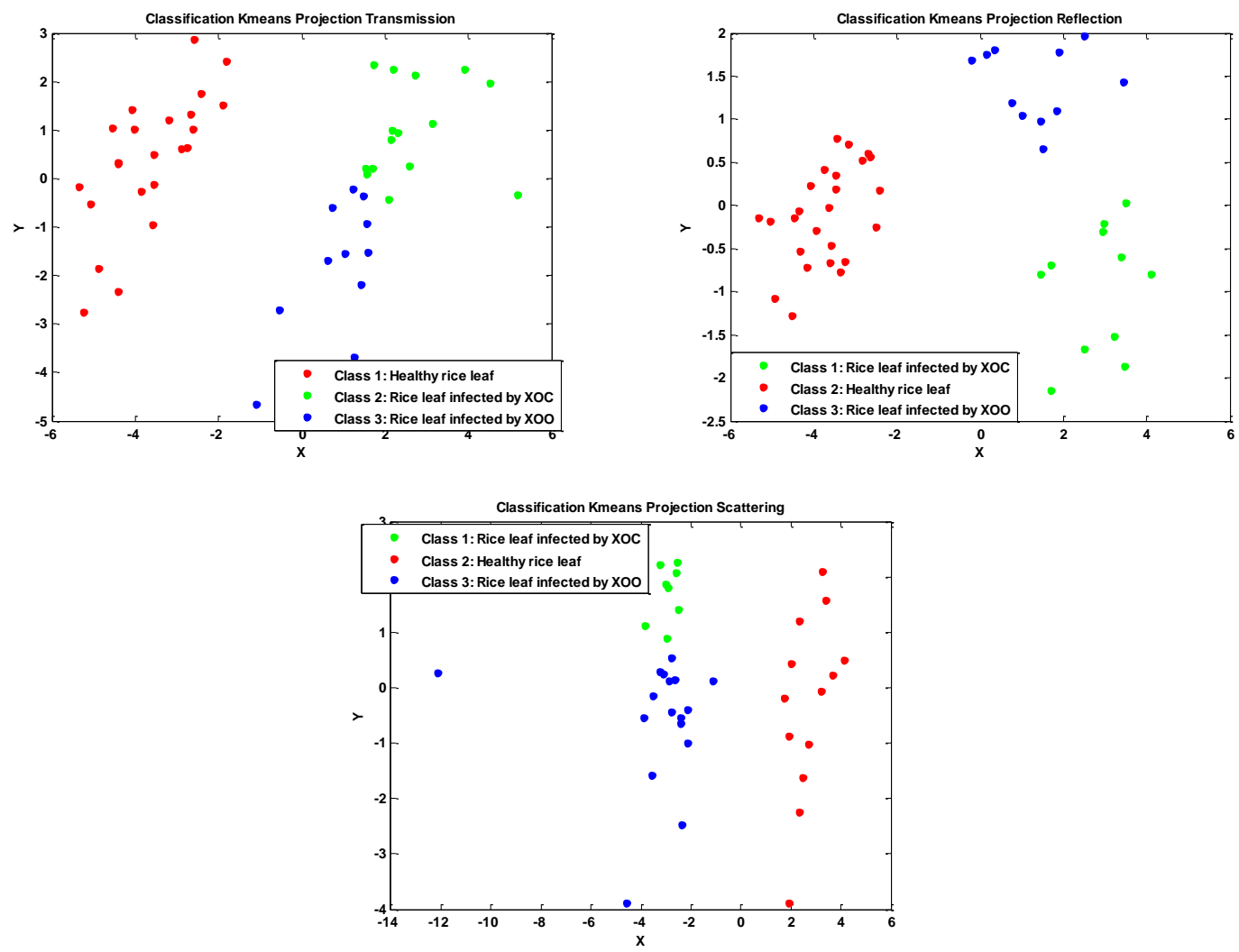

Figure 6. Projection of healthy, infected by XOO and XOC leaves in transmission (a), reflection (b) and scattering (c) modes, using K-means
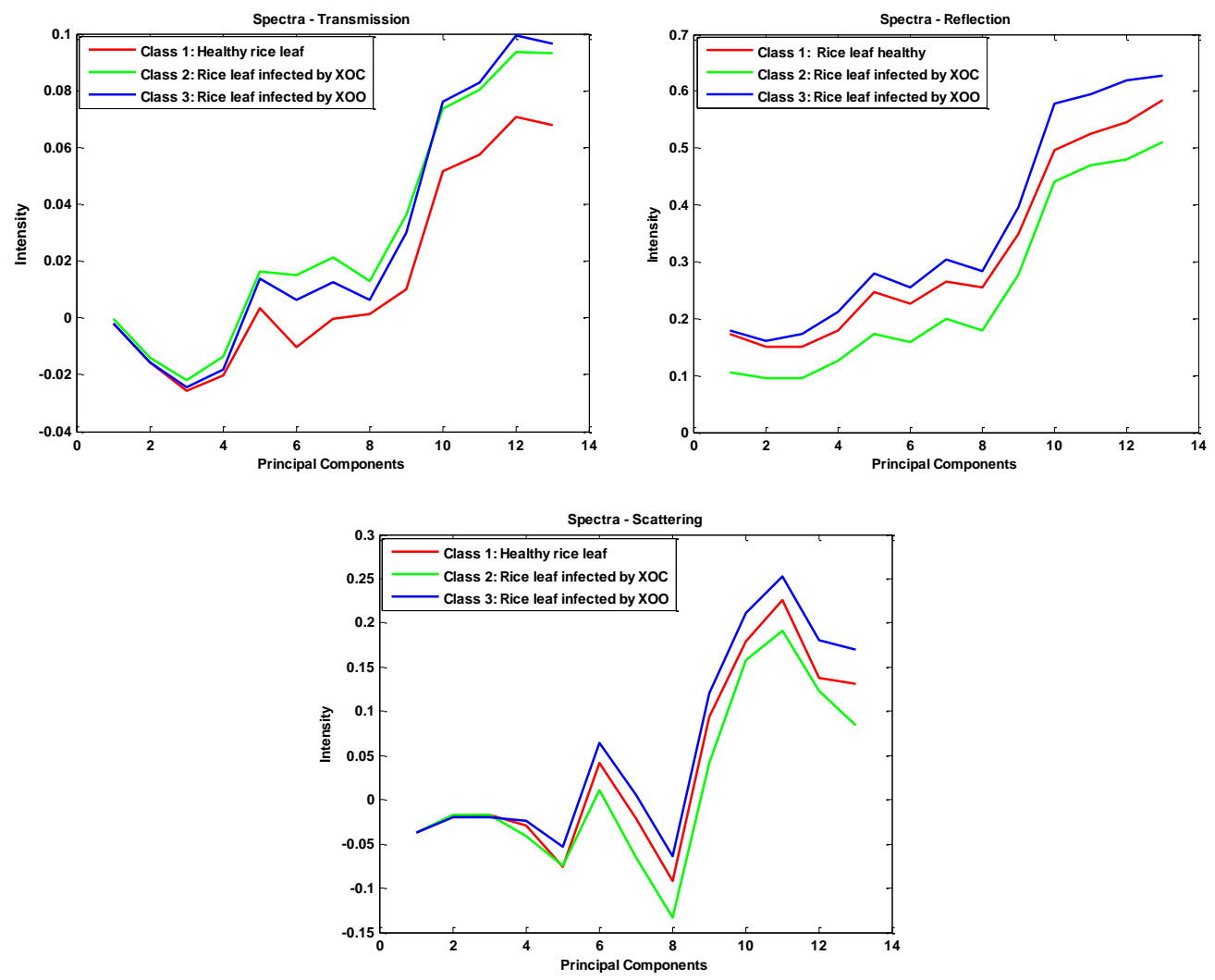

Figure 7. Spectral representation of the different classes 1, 2 and 3 in transmission (a), reflection (b) and scattering (c) modes 
The result of this classification method gives us three distinct elements in transmission, reflection and scattering namely red (healthy leaf), green (leaf infected by XOC), blue (leaf infected by XOO). Finally, three distinct classes are obtained (Figure 7) after the analysis of their optical spectra means.

\section{Discussion}

The three specimens exhibit comparable spectra shapes for the three modes; in the transmission mode, there is a low transmittance in UV and blue region, followed by a higher transmittance in the green and yellow region. The transmittance observed in the red and NIR region is lower than the ones between 500nm and $600 \mathrm{~nm}$. The highest transmittance is observed beyond $750 \mathrm{~nm}$. This is consistent with the transmittance spectra of the leaves contents, and mainly the chlorophylls ones. The reflection and scattering spectra are similarly consistent with the ones generally observed in the leaves, and exhibit a comparable shape with the transmittance. However, healthy rice leaves exhibit lowest transmittance around $400 \mathrm{~nm}$ and highest ones around $530 \mathrm{~nm}$ and after $700 \mathrm{~nm}$ compared to the infected ones; though, it reflects largely more amount of light and shows lower diffusion comparing to the infected ones. Studies have proven that the amount of reflected light depends on what has been absorbed by the plant leaves. The green color of the leaf depends on absorption degree of red light to provide the photosynthesis reaction, while the near infrared ray is reflected or transmitted fully (BOUSQUET, 2007). In this case, the leaves show a higher absorption, in chlorophylls (a and b) absorption bands, of UV and red-NIR region; the higher absorption correlates well with the photosynthesis that's happening in the presence of light radiation. So its chlorophyll rate is sufficient to receive and transform the solar energy in certain nutrients for normal growth of the rice plant (Lichtenthaler, 1987; Motte, 2007).

Even if the shapes of the leaves spectra are largely comparable, three separated classes can be observed according to the mean spectra in each mode; comparing healthy and infected leaves, we can notice basically a higher absorbance for the healthy leaves; this is consistent with better photosynthesis activities, as healthy indicator of plants. The infected leaves exhibit a lower absorbance in the region of $525 \mathrm{~nm}$ to $660 \mathrm{~nm}$, which belongs the pigments absorbance peaks. The healthier the leaves are, the higher the absorbance is. The discrimination between the XOC and XOO infection is revealed at $525 \mathrm{~nm}$ where the XOO infection exhibits a lower absorbance, and higher from $700 \mathrm{~nm}$ to $940 \mathrm{~nm}$. In reflection mode, the healthy leaves exhibit higher absorbance compared to the infected ones; this is consistent with what has been noticed in transmission. While comparing the XOO and XOC infection, the significant change is seen at $590 \mathrm{~nm}$ where XOO shows a lower reflectance. For the scattering mode, the mean spectra of healthy cells display typically lower values. The healthier the leaves are, the lower the leaves contents scatter the light. The reason is that the healthier the cells, the more homogeneous the leaves contents are. In this mode XOO infection is differentiated by higher scattering in the region of $750 \mathrm{~nm}$ to $940 \mathrm{~nm}$. The differentiation between XOO and XOC infection could result from competitive and complex activities related to the specific path in the photosynthetic level for each type of contamination. The lower absorbance for XOC infected leaves shows that it can obviously contain insufficient concentration of chlorophylls (a and $b$ ) for photosynthesis, in order to produce a small amount of glucose and oxygen to feed the rice plant. Spectral studies also reveal that the near infrared radiation (between $850 \mathrm{~nm}$ and $940 \mathrm{~nm}$ ) is not fully reflected or transmitted by these leaves. In this case, the effect of strain XOC affects the coloration of rice leaf causing yellowing and lesion on the limb. So, leaf streak caused by this Xanthomonas oryzae pv. Oryzicola strain may limit the growth and yield of rice.

The rice leaves infected by XOO absorbs and diffuses less UV lights (375 nm) and red lights $(660 \mathrm{~nm})$; but it transmits almost entirely in the visible range $(525 \mathrm{~nm})$ and near infrared $(700-940 \mathrm{~nm})$. By cons, it does not reflect the light radiation from ultraviolet to near infrared between $375 \mathrm{~nm}$ to $940 \mathrm{~nm}$; because the red light $(660 \mathrm{~nm})$ is not absorbed enough. Thus, the near infrared radiation is fully transmitted. Due to this infection of XOO strain, the rice leaves may have a green color produced by the chlorophylls ( $a$ and $b$ ) during photosynthesis to feed the rice plant. Consequently, the bacterial wilt caused by this strain of Xanthomonas oryzae pv. Oryzae may have no impact on the growth and productivity of rice.

The interpretation of the spectral signatures of these infected leaves by the strain Xanthomonas oryzae (XOO and XOC) demonstrates that their effects are harmful on the rice growing. But the severity of leaves streak caused by XOC has a detrimental effect on more than withered leaves by rice bacterial blight XOO. This can be explained by the fact that the biochemical compounds of withered leaves have many of their leaves streak, having spongy parenchyma (mesophyll) less developed. Indeed, the narrowing of this layer induces possibly, a lower exchange between oxygen and carbon dioxide, in the process of photosynthesis and respiration. Consequently, despite the attack of bacterium, all those leaves participate in photosynthesis in some way to supply to the nutritional needs of the rice plant. In addition, according to the culture environment, rice farmers can probably have an acceptable harvest. 


\section{Conclusion}

Our proposed approach shows that multispectral and multimodal microscopy is a highly qualified method for the distinction of effects caused by bacterial wilt (BLS) and leaf streak (BLB) affecting the growth and yield of rice. It allowed us to characterize the reaction of these strains according to their difference and their resemblance, and assess their rate of chlorophyll through their spectral variation depending on light intensity transmitted, reflected and scattered. This study shows that the influence of these infected leaves depend of their optical properties and the medium. The optical and spectral analyses coupled with multivariate statistical methods, of these spectroscopic images, provide powerful means to characterize the biochemical contents of leaves samples in a wide variety of applications. Furthermore, these results can be useful as a basis for the identification of such bacterial diseases in Africa.

\section{Acknowledgement}

We would like to thank the International Science Programme (ISP) for financing the project. We also thank Prof. S. Svanberg, Dr. M. Brydegaard, Mr. A. Merdasa for their technical support and Mr. Cheick TEKETE of the Laboratory of Applied Molecular Biology / Faculty of Science and Technology / University of Science \& Technology in Bamako (USTTB) Mali, for providing the samples.

\section{References}

Abdi, H., \& Williams, L. J. (2010), Principal component analysis. WIREs Comp Stat, 2, 433-459. http://dx.doi.org/ 10.1002/wics.101

Adhikari, T. B., Mew, T. W., \& Teng, P. S. (1994). Progress of bacterial blight on rice cultivar carrying different Xa genes for resistance in the field. Plant Disease, 78, 73-77. http://dx.doi.org/10.1094/PD-78-0073

Bagui, O. K., \& Zoueu, J. T. (2014). Red Blood Cells Counting by Circular Hough Transform Using Multispectral Images. Journal of Applied Sciences, 14(24), 3591-3594. http://dx.doi.org/10.3923/jas.2014

Bagui, O. K., Yavo, W., Tano, D., \& Zoueu, J. T. (2014). Etude de l'effet de l'amodiaquine sur les globules rouges infectés par le paludisme dans les images multispectrales. Afrique SCIENCE, 10(4), 36-44.

Basso A, (2005). Rice Bacterial Leaf Blight in West Africa: Preliminary studies on disease in Farmers Field and Screening released Varieties for Resistance to the bacteria. Asian Journal of Plant Sciences, 4, 577-579.

Basso, A., Onasanya, A., Issaka, S., Sido, A. Y., Haougui, A., Adam, T., ... \& Saadou, M. (2011). Le flétrissement bactérien du riz au Niger: diversité pathologique d'isolats collectés sur les périmètres irrigués. Journal of Applied Biosciences, 38, 2551-2563.

Besse, P. C. (1992). PCA stability and choice of dimensionality. Statistics \& Probability Letters, 13, 405-410.

Bousquet, L. A. (2007). Mesure et modélisation des propriétés optiques spectrales et directionnelles des feuilles (Doctoral dissertation, Paris 7).

Buddenhagen, I. W., Vuong, H. H., \& Ba, D. D. (1979). Bacterial blight found in Africa. International Rice Research Newsletter, 4, 11.

Cheema, K. K., Grewal, N. K., Vikal, Y., Sharma, R., Lore, J. S., Das, A., ... \& Singh, K. (2008). A novel bacterial blight resistance gene from Oryza nivara mapped to $38 \mathrm{~kb}$ region on chromosome $4 \mathrm{~L}$ and transferred to Oryza sativa L. Genetics research, 90(05), 397-407.

Kauffman, H. E., Reddy, A. P. K., Hsieh, S. P. Y., \& Merca, S. D. (1973) An improved technique for evaluating resistance of rice varieties to Xanthomonas oryzae. Plant Disease Reporter, 57, 537-541.

Kihupi A. L. (1997). Detection and characterization of seed-borne pathogenic bacteria of rice cultivars from Tanzania. Training report. Department of Crop Science and Production. Sokoine University of Agriculture, PO Box 3005, Morogoro. Tanzania, 64 p.

Lichtenthaler, H. K. (1987). Chlorophylls and carotenoids: pigments of photosynthetic biomembranes. Methods in Enzymology, 148, 350-382.

Marc, T. (2006). Analyse en Composante Principale comme outil d'analyse de la variabilité spectrale. P. 2, 4,10.

Merdasa, A., Brydegaard, M., Svanberg, S., \& Zoueu, J. T. (2013). Staining-free malaria diagnostics by multispectral and multimodality light-emitting-diode microscopy. Journal of biomedical optics, 18(3), 036002-036002. http://dx.doi.org/10.1117/1.JBO.18.3.036002

Mew, T. W., Vera Cruz, C. M., \& Reyes, R. C. (1992). Interaction of Xanthomonas campestris pv. oryzae and a resistance Rice Cultivar. Phytopathology, 72, 786-789. 
Motte Patrick. (2007). La photosynthèse (pp. 1-7).

Olivier, K., Bagui, J., Zoueu, T., \& Wählby, C. (2015). Automatic malaria diagnosis by the use of multispectral contrast imaging. Journal of Physical Chemical News, 75, 86-98.

Reimers, P. J., \& Leach, J. E. (1991). Race-specific resistance to Xanthomonas oryzae pv. oryzae conferred by bacterial blight resistance gene Xa-10 in rice (Oryza sativa) involves accumulation of a lignin-like substance in host tissues. Physiological and Molecular Plant Pathology, 38(1), 39-55. http://dx.doi.org/10.1016/S08855765(05)80141-9.

Sangare, M., Bagui, O., Traore, I., Babana, A., Ba, A., \& Zoueu, J. (2015). Discrimination de différentes sous phénotypes du Ralstonia Solanacearum dans une feuille de tabac par imagerie multi-spectrale. Afrique Science, 11(4), 95 - 103. Retrieved from http://www.afriquescience.info

Séré, Y., Onasanya, A., Verdier, V., Akator, K., Ouedrago, L. S., ... d Ou, S. H. (1985). Rice Diseases. Commonweath Mycological Institute Kew, Survey, England.

Shekawat, G. S., Srivastava, D. N., \& Rao, Y. P. (1969). Seed infection and transmission of bacterial leaf streak of rice. Plant Disease Reporter, 53, 115-116.

Srivastava D. N., \& Rao, Y. P. (1964). Seed transmission and epidemiology of the bacterial blight disease of rice in North India. Indian Phytopathology, 17, 77-78.

Zoueu, J. T., \& Zan, S. G. T. (2012). Trophozoite stage infected erythrocyte contents analysis by use of spectral imaging LED microscope. Journal of Microscopy, (245), 90-99.

Zoueu. J. T., Ouattara, S., Touré, A., Safi, S., et Zan, S. T. (2009). Spectroscopic approach of multispectral imaging of plasmodium falciparum infected human erythrocytes. IEEE proceedings, ICTON Mediterranean Winter Conference, 23, 1-7.

\section{Copyrights}

Copyright for this article is retained by the author(s), with first publication rights granted to the journal.

This is an open-access article distributed under the terms and conditions of the Creative Commons Attribution license (http://creativecommons.org/licenses/by/3.0/). 